\title{
Pervaporation Dehydration of Ethanol Using Nanocomposite Membranes Prepared from Poly(vinyl alcohol) and Poly(vinylpyrrolidone) Blends Incorporated with Cellulose Nanocrystals
}

\author{
SEEMA S. PATTANSHETTI and GAVISIDDAPPA S. GOKAVI* \\ Department of Chemistry, Shivaji University, Kolhapur 416 004, India \\ gsg_chem@unishivaji.ac.in
}

Received 9 February 2017 / Accepted 2 March 2017

\begin{abstract}
Pervaporation dehydration of ethanol was investigated using blend nanocomposite membranes of poly(vinyl alcohol) and poly(vinylpyrrilidone) incorporated with varying amounts of cellulose nanocrystals (CNs) as filler particles. The membranes thus prepared were cross-linked with glutaraldehyde and investigated for their PV performances as a function of filler concentration, feed water composition and temperature. The membrane characterization was done using techniques like SEM and contact angle measurements to understand their morphology and sturdiness during the PV operations. Among all the membranes tested, the membrane containing $8 \mathrm{wt} \% \mathrm{CNs}$ offered optimum values of separation factor (23976) with a reduced flux of $0.563 \mathrm{~kg} / \mathrm{m}^{2} \mathrm{~h}$ compared to that of the nascent membrane. Detailed explorations were done on the membrane that offered optimum values of PV performance in order to understand the effect feed water composition. Sorption and diffusion along with PV results were analyzed using thermodynamic models.
\end{abstract}

Keywords: Pervaporation, Cellulose nanocrystals, Diffusion, Sorption, Blend, Ethanol-water mixture

\section{Introduction}

Aqueous ethanol solutions result during fermentation processes of the disposed biomass, wherein ethanol is used as a renewable source of energy to replace conventional petroleum products. Purification and concentration of aqueous ethanol in these processes are typically carried out by distillation. However, this conventional approach is difficult to separate azeotropic compositions of water-ethanol mixture. In this pursuit, membrane-based pervaporation $(\mathrm{PV})$ separation is the preferred approach to dehydrate ethanol from its aqueous mixture ${ }^{1-5}$ as the method has lower energy demands and environmentally benign compared to conventional distillation. This has prompted researchers to develop a variety of nanocomposite membranes by incorporating nano-sized fillers into a polymer matrix in order to boost the separation performance. The incorporation of nanoscale materials into 
polymeric matrices results in a broad range of novel applications of the conventional polymers in PV separation as these materials offer better advantages in terms of mechanical, thermal and barrier properties compared to nascent polymeric membranes.

Cellulose is a natural biopolymer available abundantly from renewable sources and is biodegradable. It is obtained from sources like wood pulp, some bacteria algae, tunicates (a sea animal), grasses, cotton, etc. Compared to other nanomaterials, nanocrystalline cellulose is a low cost renewable material that can be proposed as a filler to develop nanocomposite membranes. Hydrolytic processes are commonly used to remove amorphous cellulose to form cellulose nanocrystals ${ }^{6}$ that are attractive as filler materials in view of their natural, renewable origins and good mechanical strengths ${ }^{7}$. Cellulose nanocrystals, being the crystalline portions of cellulose, are produced by acid hydrolysis, which breaks down the micro fibrils into elementary single crystallites to provide excellent membrane properties when incorporated into a polymeric matrix and can thus be good reinforcing fillers. This prompted us to consider nanocrystalline cellulose particles as fillers into the blend polymer system formed from poly(vinyl alcohol) (PVA) and poly(vinylpyrrolidone) (PVP) in order to boost its membrane performance during ethanol dehydration.

In this study, novel nanocomposite membranes were prepared by incorporating different amounts of cellulose nanocrystals (CNs) into blend matrix of PVA and PVP. Four different nanocomposite membranes were prepared by loading 2, 4 and $8 \mathrm{wt} . \%$ of CNs using solution casting technique along with a nascent blend membrane of PVA/PVP. These membranes were further characterized by SEM and contact angle measurements and subjected to PV separation studies at varying feed mixture composition of water-ethanol mixture and temperature. The effect of $\mathrm{CN}$ content on membrane sorption and diffusion was also investigated in the temperature $27^{\circ} \mathrm{C}$ to $70{ }^{\circ} \mathrm{C}$.

\section{Experimental}

Poly(vinylpyrrolidone) of $\mathrm{M}_{\mathrm{w}}=40,000$ was purchased from SRL, Mumbai, India, while poly(vinyl alcohol) of $\mathrm{M}_{\mathrm{w}}=125,000$, glutaraldehyde and ethanol were all purchased from S.D. fine Chemicals, Mumbai, India. Naturally available cotton linters were used for acid hydrolysis to produce cellulose nanocrystals. All other chemicals used were of reagent grade samples without any further purification. Double-distilled water was used throughout the experiments.

Cellulose whiskers extracted from cotton linters were prepared by acid hydrolysis ${ }^{8}$ performed using $64-65 \%(\mathrm{w} / \mathrm{w})$ sulfuric acid $\left(10 \mathrm{~mL} / \mathrm{g}\right.$ of cellulose) at $45{ }^{\circ} \mathrm{C}$ for about $60 \mathrm{~min}$ by arresting the reaction after diluting with cold water. The suspension was washed by centrifugation at $4900 \mathrm{rpm}$ for $15 \mathrm{~min}$ at $25^{\circ} \mathrm{C}$, which was dialyzed using a dialysis pouch. The regenerated cellulose was repeatedly washed with water until neutral $\mathrm{pH}$ and solid aggregates in the suspension were disrupted by sonication. The suspension was kept for 5 days and filtered through a Whatman 541 filter paper. The zeta average diameter of the CNs was measured by a Zetasizer laser light scattering equipment (model 3000HS, Malvern, Buntsford, U.K.), which showed the particle size of around $74 \mathrm{~nm}$.

Blend membranes of PVA/PVP were prepared by solution casting ${ }^{9}$ by separately dissolving $6 \mathrm{~g}$ of PVA or PVP in $100 \mathrm{~mL}$ of distilled water at $90{ }^{\circ} \mathrm{C}$ by mixing them in a 9:1 volume ratio. The mixture was stirred for 30 min until attainment of a homogeneous solution and filtered to remove the suspended particles. In situ crosslinking of the membrane was done by adding $0.3 \mathrm{~mL}$ of glutaraldehyde (GA) solution and the resulting solution was casted as a membrane onto a clean glass plate using a doctor's blade in a dust-free environment. The membranes thus formed were dried at ambient temperature $\left(30^{\circ} \mathrm{C}\right)$ and peeled off from the glass plate. 
Following the same protocol, the nanocomposite membranes were prepared by dispersing 2, 4 and $8 \mathrm{wt} . \%$ of $\mathrm{CNs}$ with respect to weight of the blend polymer solution. The mixture was stirred vigorously until uniform mixing from which membranes were fabricated. These are designated as: $\mathrm{CN}-0$ (nascent blend of PVA/PVP) to which $0.2 \mathrm{~mL}$ of conc. $\mathrm{HCl}$ was added as a catalyst for cross-linking. The nanocomposite membranes were designated as $\mathrm{CN}-2, \mathrm{CN}-4$ and $\mathrm{CN}-8$, respectively that contained 2, 4 and 6 wt.\% of CNs.

Feed and permeate samples were analyzed by gas chromatography (Model Ultima 2100, Netel India Ltd, Mumbai) installed with a thermal conductivity detector (TCD) by maintaining the oven temperature at $100{ }^{\circ} \mathrm{C}$, while the injector and detector temperatures were maintained at $150{ }^{\circ} \mathrm{C}$. The sample injection volume was $1 \mu \mathrm{L}$ and pure hydrogen was used as a carrier gas at a pressure of $1 \mathrm{~kg} / \mathrm{cm}^{2}$. The GC response was calibrated with the known compositions of ethanol-water mixtures and calibration factors were fed into the software to obtain the correct analysis for unknown samples.

Pervaporation (PV) unit consists of a stainless steel cell ${ }^{10}$ provided with water circulating jacket for maintaining a constant temperature. The feed mixture in the cell was stirred using a three-blade stirrer at $200 \mathrm{rpm}$ speed by applying a downstream vacuum pressure of 6 mbar using a vacuum pump (Model ED-21, Hindhivac, Bangalore, India). Effective surface area of the membrane was $26.03 \mathrm{~cm}^{2}$ and the liquid volume capacity of the PV cell was $200 \mathrm{~cm}^{3}$. Test membrane was equilibrated for $3 \mathrm{~h}$ with the feed mixture before performing the PV experiments. Permeate was collected in cold nitrogen traps and weighed on a digital microbalance of sensitivity of $\pm 0.01 \mathrm{mg}$ after attainment of ambient temperature. The concentrations of water and ethanol of the feed and permeate were estimated by gas chromatography. The PV performance of the membranes was assessed by calculating total permeation flux $(J)$ and separation factor $(\beta)$ using:

$$
J=\frac{W_{i}}{A \times t}\left(\mathrm{~kg} / \mathrm{m}^{2} \mathrm{~h}\right)
$$

Where $W_{i}$ is the weight of permeate $(\mathrm{kg}), A$ is the effective area of the membrane $\left(\mathrm{m}^{2}\right)$ and $t$ is permeation time (h).

$$
\beta=\left(\frac{P_{w}}{P_{e}}\right) \times\left(\frac{F_{e}}{F_{w}}\right)
$$

Here, $P_{e}$ and $P_{w}$ are wt. $\%$ of ethanol and water, respectively in permeate; $F_{e}$ and $F_{w}$ are wt. $\%$ of ethanol and water in the feed mixture. Static contact angles between water droplet and membrane were measured by contact angle meter (Rame-hart, Model 500-F1, USA) at $27^{\circ} \mathrm{C}$. Surface morphology of the membranes was assessed by environmental scanning electron microscopy (ESEM, Philips XL 30 ESEM-FEG) at an accelerating voltage of $10 \mathrm{kV}$.

Equilibrium swelling was performed gravimetrically at $27{ }^{\circ} \mathrm{C}$ on circularly cut membrane sample $(3 \mathrm{~cm}$ dia). Dry samples were weighed on a single-pan digital microbalance (model AE 240, Mettler, Switzerland) and these were placed in water-ethanol mixtures (vol. $20 \mathrm{~cm}^{3}$ ) containing 10, 15, 20 and $25 \mathrm{wt}$. $\%$ of water at $27{ }^{\circ} \mathrm{C}$ in airtight test bottles. Swelling was also measured in pure water and ethanol. Test bottles were transferred to oven maintained constant at $27{ }^{\circ} \mathrm{C}$ for $48 \mathrm{~h}$. The swollen membrane samples were weighed immediately after blotting off the liquid droplets by pressing between the filter paper wraps and \% equilibrium swelling, $D S$ was calculated as: 


$$
\text { Equilibrium Swelling }(\%)=\left(\frac{W_{s}-W_{d}}{W_{d}}\right) \times 100
$$

Where $W_{s}$ and $W_{d}$ are weights of swollen and dry membranes, respectively. The sorption selectivity, $\alpha_{s}$ was calculated as:

$$
\alpha_{s}=\left(\frac{M_{w}}{M_{e}}\right) \times\left(\frac{F_{e}}{F_{w}}\right)
$$

Where $M_{w}$ and $M_{e}$ are, respectively the mass fraction of water and ethanol in the membrane; $F_{w}$ and $F_{e}$ are, respectively for water and ethanol in the feed mixture. Diffusion selectivity, $\alpha_{d}$ was calculated using the solution diffusion theory ${ }^{11}$.

$$
\alpha_{d}=\left(\frac{\alpha_{i j}}{\alpha_{s}}\right)
$$

Here, $\alpha_{i j}$ and $\alpha_{s}$ refer to real selectivity and sorption selectivity, respectively. Considering the volatile nature of ethanol, sorption experiments were repeated thrice and the results obtained within $\pm 3 \%$ of standard errors were considered for further analysis.

Membrane density was measured by benzene displacement method. Initially, empty and benzene filled specific bottles were weighed and then a weighed quantity of the membrane was introduced into benzene-filled bottle. Excess benzene was wiped out using a soft tissue paper to measure the weight of bottle plus benzene and the membrane. Density of the membrane was calculated from the difference in weights and volume of the bottle.

\section{Results and Discussion}

Typical SEM cross-section images of CNs loaded blend nanocomposite membranes (CN-2, $\mathrm{CN}-4$ and $\mathrm{CN}-8)$ compared with that of the nascent blend membrane $(\mathrm{CN}-0)$ in Figure 1 suggests somewhat a homogeneous distribution of filler nanoparticles with no agglomeration in the case of $\mathrm{CN}-8$. However, smooth somewhat fractured surfaces with a rough texture are observed for the nanocomposite membranes. As can be seen in all the SEM photographs, homogenous mixing of CNs in the bulk of the blend polymer phase might have facilitated high water transport for the nanocomposite membranes than the nascent membrane due to the restricted transport of less polar ethanol than water.

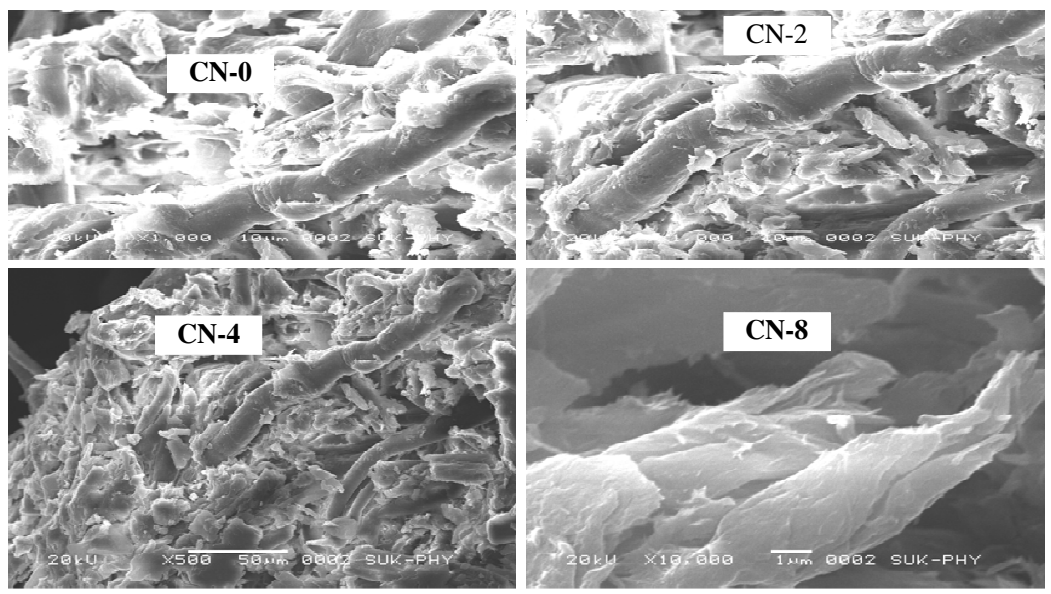

Figure 1. SEM images of cellulose nanocrystals 
Realizing that surface property of the membrane reflects its PV performance and in order to quantify whether the membrane surface acquired higher hydrophilicity at higher concentration of $\mathrm{CNs}$, we have measured water contact angle, $\theta$ that represents surface tension of a liquid and a solid in addition to interfacial tension. The $\theta$ values measured (Table 1) between water droplet and membrane surface show increasing trends with increasing CNs concentration, indicating increase of membrane hydrophobicity.

Table 1. Pervaporation performance of membranes and contact angle

\begin{tabular}{|c|c|c|c|c|c|}
\hline \multirow{2}{*}{ Membrane } & \multicolumn{2}{|c|}{ Degree of swelling, \% } & \multirow{2}{*}{$\begin{array}{c}\text { Contact angle } \\
(\theta)\end{array}$} & \multirow{2}{*}{$\begin{array}{l}\text { Flux J } \\
\mathrm{kg} / \mathrm{m}^{2} \mathrm{~h}\end{array}$} & \multirow{2}{*}{$\begin{array}{c}\text { Separation } \\
\text { factor } \beta_{\mathrm{ij}}\end{array}$} \\
\hline & Water & Ethanol & & & \\
\hline $\mathrm{CN}-0$ & 97.12 & 9.72 & 83 & 0.740 & 1666 \\
\hline $\mathrm{CN}-2$ & 96.57 & 8.27 & 89 & 0.706 & 10885 \\
\hline $\mathrm{CN}-4$ & 87.10 & 6.99 & 97 & 0.672 & 17118 \\
\hline $\mathrm{CN}-8$ & 81.06 & 5.42 & 104 & 0.563 & 23976 \\
\hline
\end{tabular}

Table 1 displays the flux and separation factor values at azeotropic mixture composition (96 wt.\% ethanol + 4 wt.\% water) at ambient temperature along with contact angle. The data shows a systematic decrease in flux at increasing loading of CNs as in case of CN-2, CN-4 and $\mathrm{CN}-8$ compared to $\mathrm{CN}-0$ membrane. This is due to increased interaction of polymer chains with water giving higher separation factor with a decrease in flux. At high filler loading, the membrane becomes rigid, causing a reduction in free volume, thereby resulting in a reduced flux. It is also noticed that with increasing temperature, separation factor values increase, but the flux values decrease.

For azeotropic mixture with $\mathrm{CN}-4$ and $\mathrm{CN}-8$, separation factors are quite high i.e., 17118 and 23976, but fluxes are low viz., 0.707 and $0.672 \mathrm{~kg} / \mathrm{m}^{2} \mathrm{~h}$, respectively (Table 1). In the case of CN-8, a further decrease in flux of $0.563 \mathrm{~kg} / \mathrm{m}^{2} \mathrm{~h}$ with the highest separation factor of 23976 is observed, possibly due to hydrophilic nature of the membrane, since the amorphous region of the blend matrix tend to decrease the permeation flux at increasing $\mathrm{CNs}$ concentration as a result of increase in amorphous region of the polymer. At high concentration of $\mathrm{CNs}$, the separation factor increases considerably due to the possible change in pore texture of the membrane, which might help to create more tortuous pathways through the available voids in the membrane. In comparison to $\mathrm{CN}-2$, higher separation factor values are observed for $\mathrm{CN}-4$ and still higher values for $\mathrm{CN}-8$.

The effect of feed water composition on PV performance was studied and it was found that a decrease in flux values with increasing concentration of CNs, while separation factor increases considerably compared to the pristine membrane. As the feed composition of water increase from 4 to $25 \mathrm{wt} . \%$, flux decreases from 0.741 to $0.563 \mathrm{~kg} / \mathrm{m}^{2} \mathrm{~h}$, while separation factor increases considerably. In case of $\mathrm{CN}-8$ membrane, the highest separation factor of 23976 is observed, suggesting that $\mathrm{CN}-8$ is most efficient for ethanol dehydration considering even at low feed water concentration.

In a PV process, permeation flux and selectivity are dependent on membrane swelling that occurs due to the sorption phenomenon as a result of interactions between membrane and transporting liquid molecules. Equilibrium swelling (sorption equilibrium) of the membranes was measured and these data at ambient temperature are shown in Table 1 . The data of Table 1 show higher equilibrium swelling with respect to water than ethanol, due to increased interactions between water and $\mathrm{OH}$ groups of the membrane that are capable of forming H-bonds with water molecule. These interactions seem to be prominent for water at which amorphous regions of the membrane may be highly swollen such that polymer chains become more flexible. This would facilitate easy transport of water molecules through membrane, but the presence of CNs might decrease the flux and increase the separation factor. 


\section{Conclusion}

We have developed novel CNs-loaded PVA/PVP blend membranes by solution casting method and employed in pervaporation dehydration of ethanol. Membrane performance was enhanced at the highest loading of $8 \mathrm{wt} . \%$ of CNs, suggesting their successful application in PV dehydration at ambient temperature and azeotropic composition. These data also support the earlier literature findings of increase in separation factor with a decrease in flux.

\section{Acknowledgement}

The authors acknowledge the financial assistance form UGC (F. No. 37-336/2009(SR)).

\section{References}

$1 \quad$ Kalyani S, Smitha B, Sridhar S and Krishnaiah A, Desalination, 2008, 229(1-3), 68-81; DOI:10.1016/j.desal.2007.07.027

2 Amnuaypanich S and Kongchana N, J Appl Polym Sci., 2009, 114(6), 3501-3509; DOI:10.1002/app.30836

3 Huang R Y M, Pervaporation Membrane Separation Processes, Elsevier Science Publishers, Amsterdam, Netherlands, 1991.

4 Zhang X H, Liu Q L, Xiong Y, Zhu A M, Chen Y and Zhang Q G, J Membr Sci., 2009, 327(1-2), 274-280; DOI:10.1016/j.memsci.2008.11.034

5 Ma Y, Wang J and Tsuru T, Sep Purif Tecnol., 2009, 66(3), 479-485; DOI:10.1016/j.seppur.2009.02.005

6 Ranby B G, Disc Faraday Soc., 1951, 11, 158-164; DOI:10.1039/DF9511100158

7 Chang, C, Zhou L, Zhang J, L and Kennedy J F, Carbohydr Polym., 2010, 82(1), $122-$ 127; DOI:10.1016/j.carbpol.2010.04.033

$8 \quad$ Naidu B V K and Aminabhavi T M, Ind Eng Chem Res., 2005, 44(19), 7481-7489.

9 Aminabhavi T M and Naik H G, J Appl Polym Sci., 2002, 83(2), 244-258; DOI:10.1002/app.2240

10 Flory P J, Principles of Polymer Chemistry, Cornell University Press, Ithaca, New York, 1953.

11 Lu P and Hsieh Y, Carbohydr Polym., 2010, 82(2), 329-336; DOI:10.1016/j.carbpol.2010.04.073 